\title{
Study on Oral Materials of the Folk Artisan of Lantern Drama in Gongxian County, South Sichuan
}

\author{
Li Jiang ${ }^{1, *}$ Maotao Zhang ${ }^{2}$
}

\author{
${ }^{1}$ Aba Teachers University, Aba, Sichuan 623002, China \\ ${ }^{2}$ Composition Department, Sichuan Conservatory of Music, Chengdu, Sichuan 623002, China \\ *Corresponding author. Email: 1046290187@qq.com
}

\begin{abstract}
Gongxian County is located in the south of Sichuan Province, at the junction of Yunnan, Guizhou, Sichuan, and Chongqing. It has a splendid history and culture and colorful traditional culture. Therefore, the lantern drama of Gongxian County was born under such a regional environment. According to the unique musical and artistic characteristics and historical and cultural characteristics of local folk songs, interview method and data sorting method are used to conduct interviews with local folk artists. The music text of the lantern drama in Gongxian County is comprehensively analyzed and sorted. Through sorting and analysis, it can be known that the folk songs in Gongxian County have the commonalities of local folk songs in China and the characteristics of regional development. However, with the impact of modern culture, its inheritance and development have been on the hazard. Its research activities are of great significance to the inheritance and development of local folk songs and to respond to the government's call for the development of local culture. This paper conducts field surveys in Gongxian County, takes the lantern drama in Gongxian County as the research object, organizes the collected audio data into music score of Chinese operas and text data, and analyzes its music text from various aspects such as music structure, so as to learn about the musical characteristics of lantern drama in Gongxian County.
\end{abstract}

\section{Keywords: Gongxian County, lantern drama, song analysis}

\section{INTRODUCTION}

According to "The Records of Gongxian County" (old records) in the period of Emperor Guangxu of the Qing Dynasty, "Gong County was originally the place where the southwestern minority groups in ancient times were conquered. After the Qin State wiped out the Kaiming State, the Bo people lived here, and then it was called Bo State". It has always been the place where ethnic minorities lived. Since the beginning of the Han Dynasty, it was under the jurisdiction of the central government, and Nanguang County was established in the sixth year of Jianyuan of Emperor Wu of Han Dynasty. In the fifth year of Kaiguang in Yuan Dynasty, the government of Qianwei moved to Fujiaba, Gongxian County from Zunyi, Guizhou Province. Since then, more Han people have moved in. In the second year of Yonghe of Emperor $\mathrm{Mu}$ of the Eastern Jin Dynasty, Li Shi, the last emperor of the Han Dynasty, introduced Liao people from Yilong to Sichuan. The number of Liao people in Gongxian County also increased greatly. During the reign of Emperor Wu of Han Dynasty, Lexing, one of the major ethnic groups, occupied Ningzhou, and then Gongxian was abandoned. Many "Wuman" also known as (Yi nationality) entered Gongxian. In Song Dynasty, some Miao people moved in slowly, which was called "Nanguang Barbarians". In this way, Gongxian County became a place where Han nationality, Miao nationality, Yi nationality and Liao nationality lived together. Influenced by local culture, topography and environment, Gongxian lantern drama has developed its unique regional color and artistic characteristics. This paper will make the analysis of local music, music score from the perspective of music text, which is of important value for better inheritance of local culture.

\section{ANALYSIS OF GONGXIAN LANTERN DRAMA "BAIXIANGDIAO"}

The lantern drama in Yibin area is a song that combines singing, dancing and performance in some fixed festivals. The content of the drama is rich, including singing local historical stories, myths and legends, farming season, love and so on. The drama covers a wide range of contents, and the performance form is novel. It is generally loved by the local people. The local lantern drama has certain regional characteristics, and the mode, tonality, melody, song lyrics of the drama have certain rules can be found, but 
also have certain local characteristics. This paper will make the analysis on local lantern drama combining with the collection of music score data.

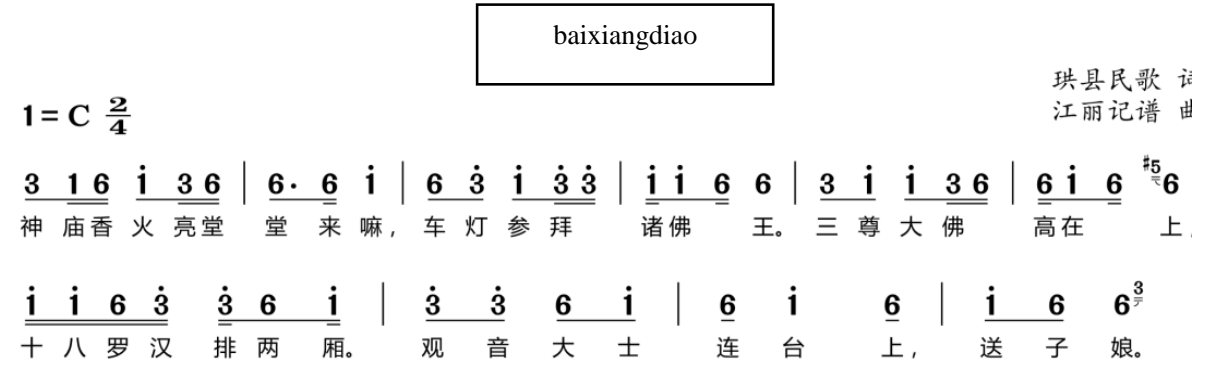

Fig. 1. The music score of "baixiangdiao".

As shown in "Fig. 1", from the perspective of lyrics, the content shows that on the way to play the lantern, the local people pass through the temple. According to the local custom, they must sing the lantern drama related to it to show their respect for the Bodhisattva, and pray to the Bodhisattva for good weather in the coming year according to the custom of singing lanterns. From the literary perspective, the lyrics describe the scene of singing lantern and playing lantern in Yibin, Sichuan Province. And the lyrics mainly take two rhythms of a and ang as the end. In addition, Yibin's local dialect singing makes the description scene vivid. From the perspective of the rhyme of the lyrics, the unique dialect melody trend and pitch sense contribute to the formation of the melody of the folk song "baixiangdiao", and finally the catchy melody and single popular lyrics are the main features of the song. As shown in "Table I":

TABLE I. THE MUSICAL FORM STRUCTURE OF "BAIXIANGDIAO"

\begin{tabular}{|l|l|l|l|l|}
\hline & a & b & c & d \\
\hline & opening & developing & changing & concluding \\
\hline number of bars & 2 & 2 & 3 & 3 \\
\hline $\begin{array}{l}\text { Structure } \\
\text { combination }\end{array}$ & 2 & 2 & $2+1$ & $2+1$ \\
\hline
\end{tabular}

In terms of musical form and structure, the folk song "baixiangdiao" inherits the characteristics of Chinese traditional four phrases in terms of the number of phrases. In terms of the number of bars in the music phrase, the work presents an irregular form of the structure of the front and back phrases. In particular, the "long-short" structural combination in the last two phrases is the core of music rhythm, reflecting the high unity and conciseness of music structure. This has contributed to the rich diversity of Chinese folk music.

In terms of pitch organization, this folk song is composed of la-do-mi. The whole song takes la tone as the center and the remaining two tones are transformed in the range. Finally, the maximum interval gap of the drama is controlled in the interval relationship of 10 degrees. For the melody line trend, it has formed a mirror image symmetry within the bars of the drama. (As shown in "Fig. 2") In terms of the interval relationship of the melody trend, the pre-rhythmic weak-beat interval relationship becomes the middle strong-beat interval relationship of the post-rhythm type. From the perspective of the relationship, it forms symmetry, which forms a feature of the pitch trend relationship in the melody singing of the folk song "baixiangdiao".

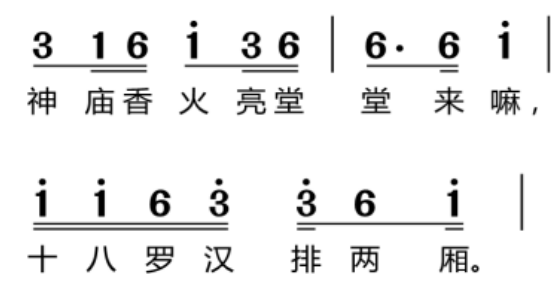

Fig. 2. Parts of music score of "baixiangdiao".

From the perspective of rhythm, the works is mainly composed of "long-short" rhythm, such as: the first eight rhythm and the sixteen rhythm later, the front small attachment, segmentation, etc. It is worth mentioning that in this kind of rhythm combination form, the music melody has a great leap in intervals of three, four, five and six. This kind of combination of long and short melodies and the switch of pitch and range in a short time undoubtedly increases the difficulty of singing. This kind of small curve and jump in melody should be completed in the short-time rhythm, showing the charm of folk songs in the region. This kind of folk song seems simple, but it is the essence of national culture. 


\section{ANALYSIS OF GONGXIAN LANTERN DRAMA "DUOXIECHA, CHENGXIECHA"}

This work is in E pentatonic mode, $2 / 4$ beats. From the perspective of melody, this work is mainly developed in the three-tone series of mi-sol-la, and the $\mathrm{mi}$ tone at the beginning and the end reveal the unity of mode and tonality. In the development of musical melody, the simple three-tone series of mi-sol-la is developed to four-tone series of mi-sol-la-re, and finally the pentatonic mode of mi-sol-la-re-do is formed. The step-by-step pitch organization is closely related to the structure, melody and music frame structure. (as shown in "Table II" and "Fig. 3")

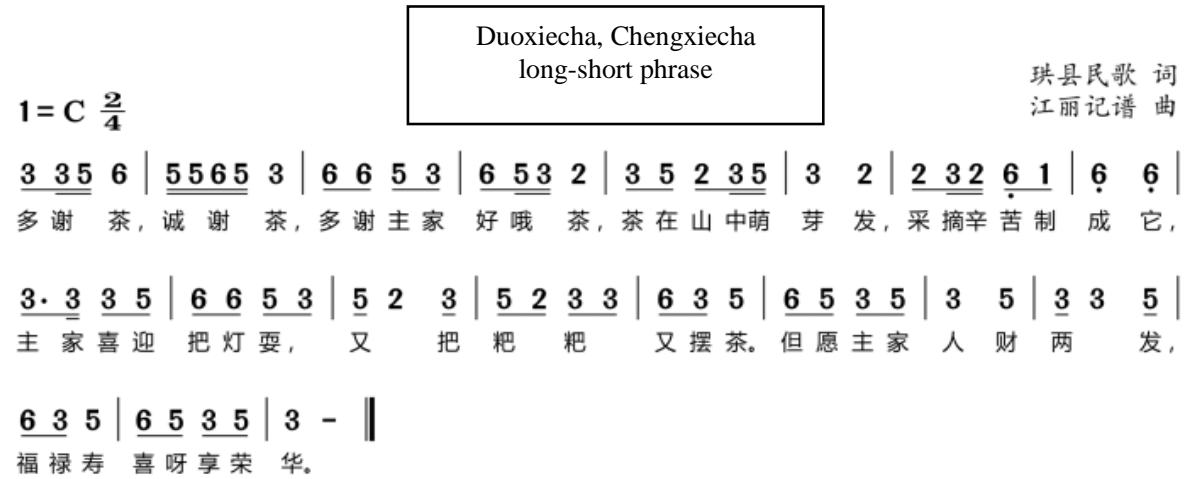

Fig. 3. The music score of "Duoxiecha, Chengxiecha".

TABLE II. MUSiCAL FORM STRUCTURE OF "DUOXIECHA, CHENGXIECHA"

\begin{tabular}{|l|l|l|l|l|}
\hline & a & b & c & d \\
\hline & opening & developing & changing & concluding \\
\hline number of bars & 4 & 4 & 5 & 6 \\
\hline phrase structure combination & $1+1+2$ & $2+2$ & $2+3$ & $3+3$ \\
\hline
\end{tabular}

In terms of the number of phrases, the folk song "Duoxiecha, Chengxiecha" retains the traditional folk song structure of four phrases, in which the relationship of opening, developing, changing and concluding is mainly based on the description of lyrics and the trend of melody. From the internal structural characteristics of the phrase, the structural combination characteristics of the works are very consistent with the expression of the lyrics. There are $1+1+2$ comprehensive structure, $2+2$ and $3+3$ periodic structure, and $2+3$ increasing structure.

Such rich changes in a folk song are inseparable from its own regional culture. For example, in the lyrics of the first phrase, "thanks for your tea, thanks for your tea, thanks for host's good tea." The rhythm of the lyrics and the comprehensive structure of the phrase $1+1+2$ are very appropriate. In the lyrics of the second phrase, "tea sprouts in the mountains, and it is hard to pick it." The arrangement of the lyrics and the position change of the rhythm make the periodic structure of phrase bar $2+2$ guaranteed when the rhythm of the same material are skillfully changed. In the lyrics of the third phrase, the length of the lyrics of "the host is glad to play with the lamp, and then cake and white tea" is changed with the sound of the previous sentence, but in the number of bars, it becomes an incremental structure of $2+3$, especially in the "又" character, the extension of the note duration increases the sense of tone of the host family, which is the same as the context of local dialect culture, showing the simplicity of this work. Finally, the lyrics of the phrase "I hope the host will have both wealth and families, happiness and prosperity". The lyrics of this phrase contrast the first two lines of lyrics, especially the word "two" becomes a long tone in melody, with blessing and exclamation. And there is also $3+3$ periodic processing of the number of bars. Through the change of lyrics, the flexibility of this folk song will enrich the structure and melody.

From the perspective of rhythm, the time value of quantifiers and auxiliary words becomes longer, forming the characteristics of one word and multi tone, which distinguishes most songs from the characteristics of one word and one sound, so as to form a strong sense of dynamic language and express thanks. (as shown in "Fig. 4"). It is worth mentioning that in the works, the subtle change of the rhythm position solves the pause and binding between the musical phrases. The strong and simple use of musical vocabulary makes people sigh at the strong artistry of Yibin folk songs in Sichuan.

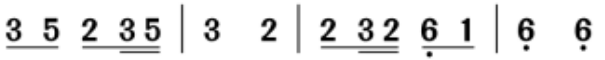

$$
\begin{aligned}
& \text { 茶在山中萌芽发, 采摘辛苦制成它, }
\end{aligned}
$$


Fig. 4. Parts of music score of "Duoxiecha, Chengxiecha".

\section{ANALYSIS OF GONGXIAN LANTERN DRAMA "CAIZI"}

In "Table III", the work belongs to E pentatonic mode, $4 / 4$ beats. The pitch melody of the whole drama is composed of la-do-mi, which is the same as the melody elements of "baixiangdiao". The pitch gap of the lowest range and the highest pitch of the drama are within an octave, and the maximum span between the two tones is a pure five degree. Such a small range span and a three-tone voice train support the whole work.

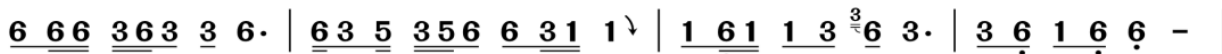

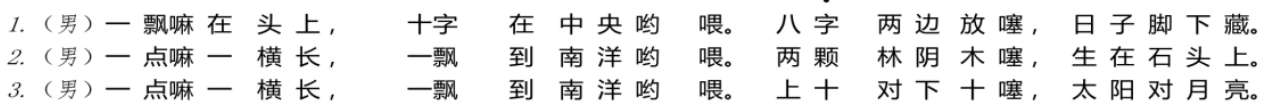

\begin{tabular}{lllllll|llllll|lllllll|llllll|}
6 & 1 & 1 & 1 & 3 & 3 & 1 & $\underline{3}$ & 1 & $\underline{3}$ & 3 & 1 & 0 & $\underline{6}$ & $\underline{6}$ & $\underline{6}$ & 3 & $\underline{6}$ & 3 & $\mid$ & $\underline{1}$ & 3 & $\underline{3}$ & 1 & 1 & -
\end{tabular}

情妹 啊! 你看是个什么字样? （女) 十八的小情哥啊, 我的小情郎。

情妹 啊! 你看是个什么字样? （女）谢拜 小情哥啊, 我的小情郎。

情妹啊! 你看是个什么字样? (女) 情妹猜不到啊, 情哥帮个忙。

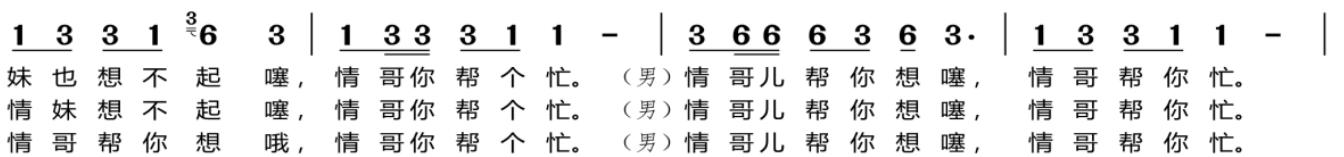

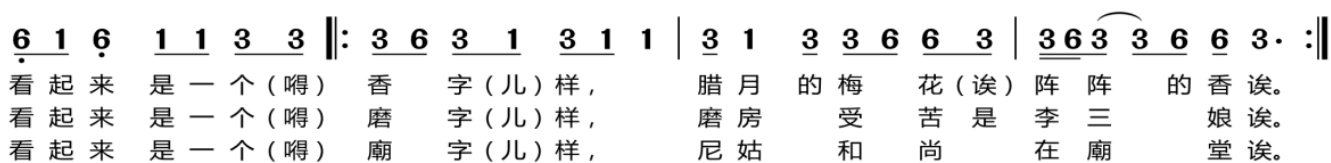

Fig. 5. Music score of "Caizi".

TABLE III. S STRUCTURAL ANALYSIS OF "CAIZI"

\begin{tabular}{|l|l|l|l|l|}
\hline \multicolumn{5}{|c|}{ musical form structure 1 of "Caizi" } \\
\hline & a & b & c & d \\
\hline & opening & developing & changing & concluding \\
\hline number of bars & 4 & 4 & 4 & 4 \\
\hline phrase structure combination & $2+2$ & $2+2$ & $2+2$ & $2+2$ \\
\hline \multicolumn{5}{|l|}{ musical form structure 2 of "Caizi" } \\
\hline & a & b & a' \\
\hline & Male voice & female voice & Male voice & \\
\hline phrase number & 3 & 2 & 3 & \\
\hline number of bars & 6 & 4 & 6 & $2+2+2$ \\
\hline phrase structure combination & $2+2+2$ & $2+2$ & & \\
\hline
\end{tabular}

In terms of musical form structure, "Caizi" is shown in "Fig. 5". This folk song reflects the diversity of music structure. Starting from the breath of the phrase, according to the traditional musical form analysis, the work still has the typical characteristics of four phrases of folk songs, and its structural integrity is more prominent. In terms of the content of the lyrics, the division of musical phrases presents the characteristics of three parts. The number of melodic phrases sung by boys at both ends is three music phrase, while the number of melody phrases sung by girls in the middle is two music phrase, forming a structural framework of $3+2+3$. The internal composition of the phrase still maintains the periodic structure characteristics.

From the perspective of rhythm, the beat of the song has changed from $2 / 4$ beats to $4 / 4$ beats, and the duration length of the song also has a longer change, which is consistent with the content of love songs. This 
kind of narrative love song not only retains the language rhythm characteristics of Yibin regional culture, but also highlights the audibility, singing and performance of music.

\section{ANALYSIS OF GONGXIAN LANTERN DRAMA "TWELVE FLOWERS"}

On the judgment of the mode and tonality of this work, it can be understood as the mode of G Qing

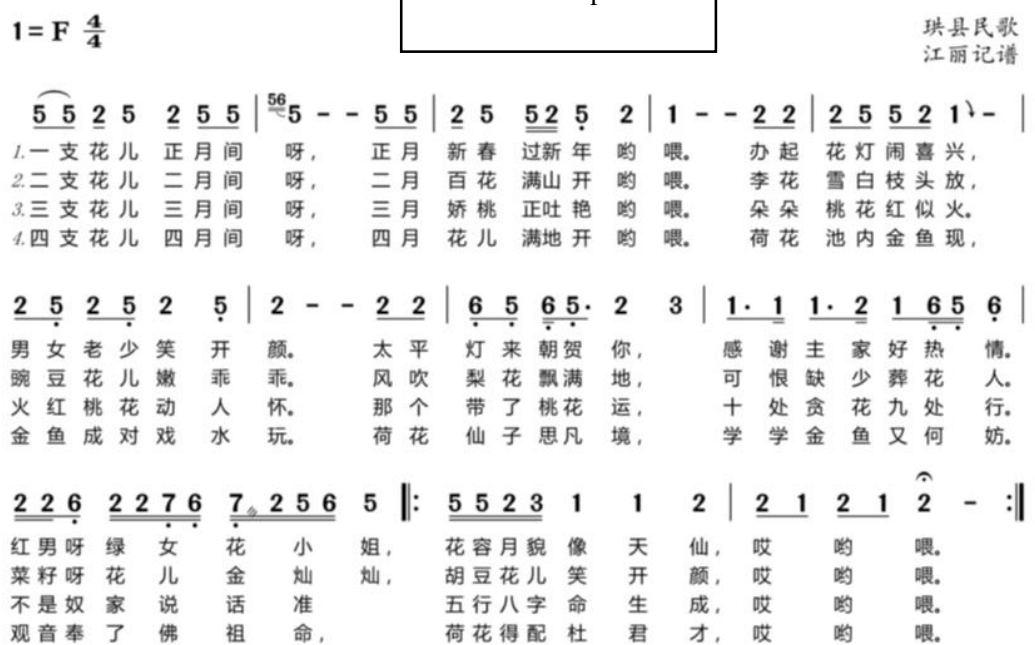

$$
\begin{aligned}
& \text { Twelve Flowers } \\
& \text { 荷花得配杜君才, 叹啲喂 }
\end{aligned}
$$

seven-tone mode. However, in the survival and development of folk music, it is by no means a mode of tonality going to the end, such diversity in folk music will be ignored. Therefore, it is required to make a detailed analysis of the work from the perspective of musical structure. (as shown in "Table IV" and "Fig. 6")

Fig. 6. Music score of "Twelve flowers".

\begin{tabular}{|c|c|c|c|c|}
\hline \multicolumn{5}{|c|}{ Analysis of the musical form of "twelve flowers" } \\
\hline & $\mathrm{a}$ & $\mathrm{b}$ & $\mathrm{c}$ & $\mathrm{d}$ \\
\hline number of bars & 4 & 3 & 2 & 3 \\
\hline Mode & F do & G re & D la & G re \\
\hline Pitch material & $\begin{array}{l}\text { three-tone series } \\
\text { (do-re-sol) }\end{array}$ & $\begin{array}{l}\text { three-tone series } \\
\text { (do-re-sol) }\end{array}$ & $\begin{array}{l}\text { five-tone series } \\
(\text { do-re-mi-sol-la })\end{array}$ & $\begin{array}{c}\text { four-tone series } \\
(\text { sol-la-xi-re }) \\
(\text { do-re-mi-sol })\end{array}$ \\
\hline re-series change & same tone & same tone & same tone & changing mode \\
\hline density of rhythm & sparse & sparse & sparse & dense \\
\hline
\end{tabular}

TABLE IV. STRUCTURAL ANALYSIS OF "TWELVE FLOWERS"

Through the analysis of the form of the folk song "twelve flowers", the number of phrases is four from the structure. From the aspect of length proportion, the work presents the characteristics of asymmetry. In the aspect of music material, the elements of pitch material of the first and second phrases are the same, belonging to the three-tone series of do-re-sol. In terms of pitch material, the third phrase inherits the same system of the first two phrases. In terms of pitch number, it changes from the original do-re-sol to do-re-mi-sol-la, forming a transformation form. In the fourth phrase, there are changes in pitch material. The number of tone series changes from the five-tone series of the previous phrase to four-tone series, changing from sol-la-xi-re to do-re-mi-sol and forming a five-degree re system transformation. Therefore, the folk song "twelve flowers" is irregular in structure. It can be seen from the analysis of pitch material that the musical structure expresses opening, developing, changing and concluding through pitch elements.

In terms of rhythm, the work belongs to $4 / 4$ beats, and the looseness of rhythm is reflected in the four phrases. The first three phrases are mostly based on long-term rhythms. From the second half of the third phrase to the fourth phrase, there are a large number of sixteen element rhythms. The intensive word-word 
rhythm in the dynamic value of music forms a small climax from loose to tight. In addition, through the change of the beats, the original regular rhythm are treated with flexibility. For example, at the beginning of the music, the lyrics are in the positive beat. After the long tone of the second bar, the intrusive weak starting bar is formed. This form of phrase remains until the sixth bar and returns to the regular rhythm of the beginning. The two ends of the fixed rhythm are transformed in the middle. It is a highlight of Gongxian folk songs in Yibin, Sichuan. This is closely related to the local customs, life and language habits. Therefore, through the analysis and interpretation of the music text, the local life form of Gongxian County in Yibin, Sichuan Province can be demonstrated.

\section{ANALYSIS ON THE ARTISTIC FEATURES OF GONGXIAN LANTERN DRAMA}

According to the analysis of the lantern drama in Gongxian above, it can be seen that the local lantern drama has the characteristics in terms of structure, tonality, lyrics content, rhythm, and pitch materials. The following are shown in "Table V".

TABLE V. MODULATION STRUCTURE ANALYSIS

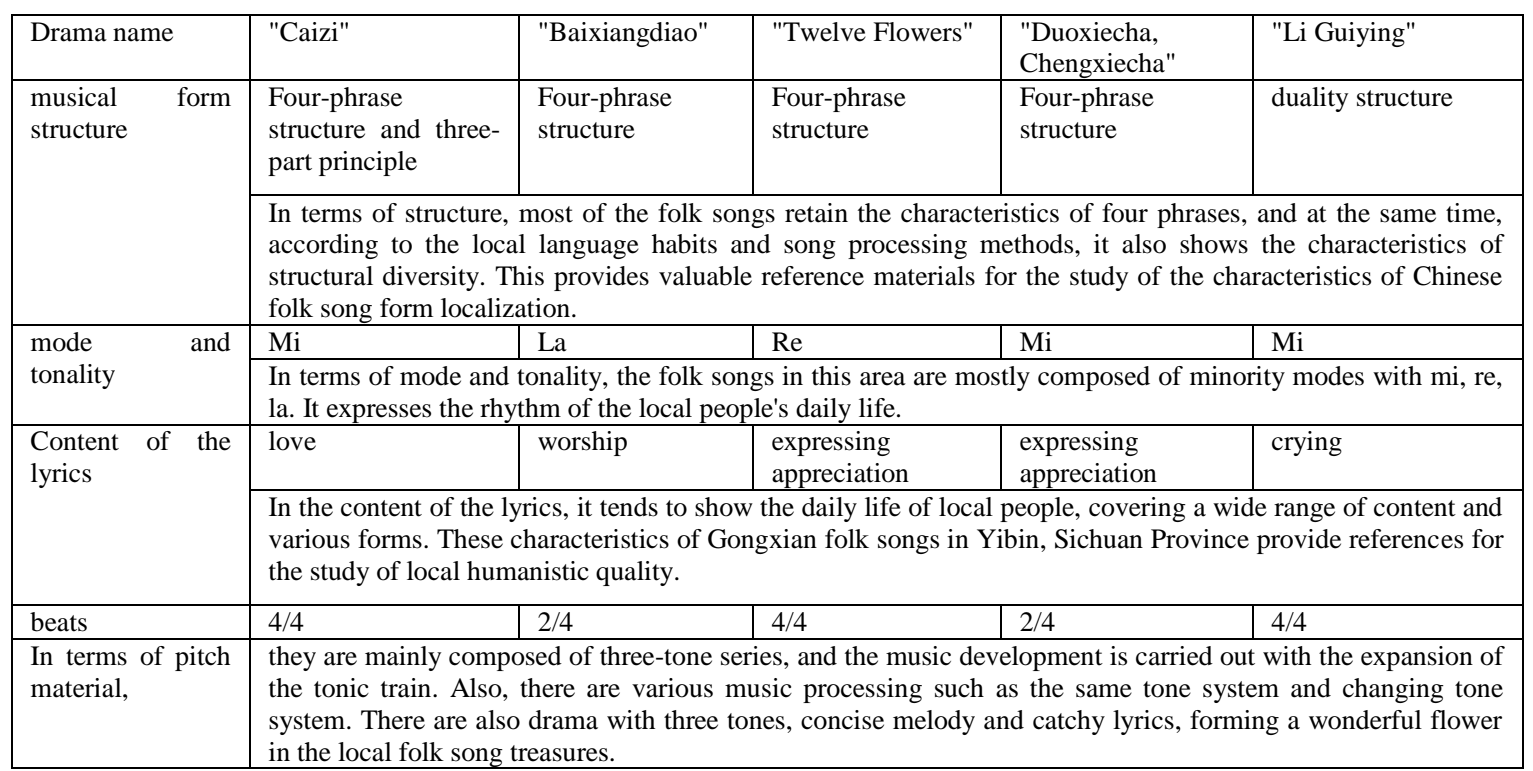

\section{CONCLUSION}

Gong County has always been inhabited by "Bo people". The powerful "Bo people" have been living here, with a lot of precious cultural influence. In addition, Gongxian has always been a place where ethnic minorities live together. Therefore, the local "lantern drama" of Han nationality are integrated with these rich historical cultures, forming the unique artistic characteristics.

Through the analysis of the local "lantern drama", it is found that the structure of the local "lantern drama" is mostly composed of four-phrase structure, duality structure, four-phrase structure and three-part principle. In structure, most of them retain the characteristics of the four phrases. Meanwhile, according to the local language habits and song processing methods, it also shows the characteristics of structural diversity. This provides valuable reference materials for the study of the characteristics of Chinese folk song form localization. Most of the local songs are based on 4/4 beats and 2/4 beats. The content of the lyrics includes: thanking the host, singing love stories, narrative singing, etc. It may be in the form of antiphonal singing, singing while dancing, singing while talking, singing while performing, etc. It can be said that the local lantern songs are very rich in content and performance form, and have certain research value. The author has interviewed with local old folk artists. Through the recording, music arrangement, people can have a certain understanding of the local lantern music, which has a certain role in further understanding Chinese local music culture, and also has a certain role in promoting local culture.

\section{References}

[1] Chen Wan. How to sing Sichuan and Chongqing folk songs [M]. Sichuan: Sichuan Literature and Art Publishing House, 2008.32-37. (in Chinese)

[2] Jiang Mingdun. Introduction to Chinese Folk Music [M] Shanghai: Shanghai Music Publishing House, 2016.40-82. (in Chinese) 
[3] Jiang Mingdun. An overview of Han folk songs [M]. Shanghai: Shanghai Literature and Art Publishing House, 1981.202-339. (in Chinese)

[4] Yang Mingkang. Chinese Folk Songs and Rural Society [M], Jilin: Jilin Education Press, 1993. 205-230. (in Chinese)

[5] Xiong Luxia. Appreciation of Chinese Classic Folk Songs [M]. Beijing: People's Music Publishing House, 2009.56-80. (in Chinese)

[6] Kuang Tianqi. A Brief Introduction to Sichuan Han Folk Songs [M], Beijing: China Drama Publishing House, 2008.102-288. (in Chinese)

[7] Huang Xiaohui. Research on Yibin Folk Song Music Culture [M], Sichuan: Sichuan University Press, 2009.20-56. (in Chinese)

[8] Zhu Jiaqi. Collected Works of Sichuan Folk Music Research [M], Sichuan: Popular Literature and Art Publishing House, 2008.40-56. (in Chinese)

[9] Xu Jiangzhen. Introduction to the Runqiang of Han Folk Songs [M], Beijing: People's Music Publishing House, 2009, 29-96. (in Chinese)

[10] Li Jiti. Chinese Music Structure [M], Beijing; Central Conservatory of Music Press, 2004, 10-84. (in Chinese)

[11] Tan Weiwei. "Study on the Artistic Features of Yunlian Folk Songs in Yibin" [D], Sichuan Conservatory of Music, 2016. (in Chinese)

[12] Lei Xiaolan. "The Characteristics and Inheritance of Ancient Songs in Southern Sichuan" [J], Contemporary Music, May 2015. (in Chinese)

[13] Mao Keqiang. "Sophora blooms in the spring - Comment on the modern Sichuan opera "When does Sophora bloom" [J], Sichuan Drama, May 2011. (in Chinese)

[14] Shen Wen. "Inheritance Status and Protection Countermeasures of automative lighting under the Vision of "Intangible Cultural Heritage" Protection" [J], Chinese National Academy of Arts, 2016-05-01. (in Chinese)

[15] Liu Yutong. A Review of the Music Research of Bo People in Yibin, Sichuan [J], Music Creation, July 2011. (in Chinese)

[16] Huang Xiaohui. "Overview of Yibin Folk Songs in Southern Sichuan" [J], Great Wall, October 2009. (in Chinese)

[17] Chen Lian. "An Analysis of Sichuan God Songs" [J], The Voice of the Yellow River, February 2015. (in Chinese)

[18] Luo Jianhua. Cool breeze, Guangming Daily Publishing House, 2007. (in Chinese)

[19] Gongxian Poetry Couplets Association, Gongxian Reading Club, 2017. (in Chinese)

[20] Luo Jianhua. Transcript of Gongxian folk songs, internal data of Gongxian, 2015. (in Chinese) 